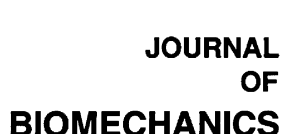

Journal of Biomechanics 40 (2007) 2483-2491

www.elsevier.com/locate/jbiomech www.JBiomech.com

\title{
Parametric geometry exploration of the human carotid artery bifurcation
}

\author{
Neil W. Bressloff* \\ School of Engineering Sciences, University of Southampton, Highfield, Southampton SO17 1BJ, UK
}

Accepted 10 November 2006

\begin{abstract}
A parametric computational model of the human carotid artery bifurcation is employed to demonstrate that it is only necessary to simulate approximately one-half of a single heart pulse when performing a global exploration of the relationships between shear stress and changes in geometry. Using design of experiments and surface fitting techniques, a landscape is generated that graphically depicts these multi-dimensional relationships. Consequently, whilst finely resolved, grid and pulse independent results are traditionally demanded by the computational fluid dynamics (CFD) community, this strategy demonstrates that it is possible to efficiently detect the relative impact of different geometry parameters, and to identify good and bad regions of the landscape by only simulating a fraction of a single pulse. Also, whereas in the past comparisons have been made between the distributions of appropriate shear stress metrics, such as average wall shear stress and oscillatory shear index, this strategy requires a figure of merit to compare different geometries. Here, an area-weighted integral of negative time-averaged shear stress, $\tilde{\tau}$, is used as the principal objective function, although the discussion reveals that the extent as well as the intensity of reverse flow may be important. Five geometry parameters are considered: the sinus bulb width, the angles and the outflow diameters of the internal carotid artery (ICA) and external carotid artery (ECA). A survey of the landscape confirms that bulb shape has the dominant effect on $\tilde{\tau}$ with maximum $\tilde{\tau}$ occurring for large bulb widths. Also, it is shown that different sets of geometric parameters can produce low values of $\tilde{\tau}$ by either relatively small intense areas, or by larger areas of less intense reverse flow.
\end{abstract}

(C) 2006 Elsevier Ltd. All rights reserved.

Keywords: Parametric geometry; Carotid artery bifurcation; Design exploration

\section{Introduction}

Combined with the cardiovascular pulse, the shapes (or geometries) of arterial bifurcations are such that the accompanying blood flow is believed to produce favourable conditions for the development of atherosclerosis. There is now some consensus for the correlations between locations of atherosclerosis and regions of low mean shear stress, high oscillation and high mean shear stress gradients (Buchanan and Kleinstreuer, 1998; Ku et al., 1985; Zairns et al., 1983). As a result, a number of studies have investigated the impact of geometrical variations on flow features and on the correlations of these load metrics with

\footnotetext{
${ }^{*}$ Tel.: + 442380595473 ; fax: + 442380592168

E-mail address: N.W.Bressloff@soton.ac.uk.
}

sites of arterial disease (Spelde et al., 1990; Perktold et al., 1991; Wells et al., 1996; Schulz and Rothwell, 2001; Sitzer et al., 2003). Inspired by much of the above research, the current work demonstrates a strategy for visualising the multi-dimensional relationships relating shear stress metrics to five carotid artery bifurcation shape parameters: the angles and outflow diameters of the internal carotid artery (ICA) and the external carotid artery (ECA) and the bulb width. It accomplishes this by constructing a surrogate model of the so-called design space that represents the relationship between the shape parameters and a shear stress metric - the area-weighted integral of negative timeaveraged shear stress denoted by $\tilde{\tau}$ and defined as

$\tilde{\tau}=\frac{\left|\sum_{i} \gamma_{i}\left(\bar{\tau}_{-}\right)_{i} \mathrm{~d} A_{i}\right|}{\sum_{i} \gamma_{i} \mathrm{~d} A_{i}}$ 
integrated across all wall mesh cell faces with incremental area $\mathrm{d} A_{i}$, where for the $i$ th element

$$
\left(\bar{\tau}_{-}\right)_{i}=\frac{\sum_{N}\left|\tau_{\mathrm{w}}\right|(-1)^{j}}{N}
$$

signifies the average value of the wall shear stress magnitude, $\left|\tau_{\mathrm{w}}\right|$, across $N$ time steps, premultiplied by -1 $(j=1)$ if the $y$-component of $\tau_{\mathrm{w}}$ is negative; for a positive $y$-component of $\tau_{\mathrm{w}}, j=0 . \gamma_{i}$ is defined as

$\gamma_{i}=\begin{array}{ll}1 & \text { if }\left(\bar{\tau}_{-}\right)_{i}<0 \\ 0 & \text { if }\left(\bar{\tau}_{-}\right)_{i}>0\end{array}$

such that the summations in Eq. (1) are only performed for cell faces on which the average shear stress defined in Eq. (2) is negative. In this way, $\tilde{\tau}$ captures the extent and intensity of shear stress in regions of reverse flow in a single scalar. Traditionally, distributions of shear stress related metrics have been presented and, indeed, the variation in $\bar{\tau}_{-}$for different bifurcations is depicted in the discussion below. Other metrics that could have been used include those recently discussed in Hyun et al. (2004). However, the key point to appreciate is that, here, a figure of merit (or objective function) is required to numerically compare the behaviour/performance of different bifurcations. Whilst an integrated quantity masks the haemodynamic effect on individual cells it is not implausible that the spatially averaged quantity $\tilde{\tau}$ (obtained from the spatial distribution of the time-averaged quantity $\bar{\tau}_{-}$, for cell faces exposed to reverse flow) could be shown to be a valid metric for comparison. Whilst still speculative at this stage, it is interesting to ponder whether endothelial cells in a particular region are more adversely affected by larger regions of less intense reverse flow compared to smaller regions of stronger recirculation.

$\tilde{\tau}$ is extracted from computational fluid dynamics (CFD) simulations performed using Fluent (Fluent Inc.) in individual shapes, each of which is produced by manipulating a parametric model of a typical bifurcation constructed in the computer aided design (CAD) package, CATIA V5 (Dassault Systemes). The computational geometries required for the current approach provide an alternative geometry construction methodology to the geometries used by many other researchers. Typically, these have been derived from digitised images of real anatomy obtained from magnetic resonance imaging and ultrasound (see, for example, Milner et al., 1998; Augst et al., 2003, respectively) or have been based on the experimental glass models described by Bharadvaj et al. (1982) and, more recently, by Ding et al. (2001). Thus, although the usefulness of this approach may not be immediately obvious, since clinicians typically deal with scanned images of real anatomy, the potential benefits are, in fact, far reaching. First, as demonstrated in this article, the relative importance of different geometric features can be quantified in a broad sense as opposed to considering a small number of discrete geometries. The systematic exploration of such relationships should prove particularly useful when analysing the haemodynamic effects associated with less well understood features. For example, preliminary work has commenced to better understand the impact of non-planar geometry in the carotid artery bifurcation (Bressloff et al., 2005) and a model is currently under construction with the intention of exploring how the shape of the divider in the bifurcation affects the flow and associated wall interactions. Second, the construction of surrogate models to encapsulate the detailed analysis of these relationships provides the framework for a very fast diagnostic procedure (significantly faster than performing full CFD simulations). This could comprise an inverse design strategy to pin-point any particular scanned anatomy within the surrogate model, and thus provide an indication of both geometric quality and ways in which it could be improved. Finally, these techniques are not limited to the carotid artery bifurcation and could be applied throughout the cardiovasculature, and in particular to stent and bypass graft design (Bhaskar et al., 2005).

\subsection{Geometry exploration}

Whereas previous work has compared a relatively small number of different bifurcation shapes, the present article describes a systematic approach that is designed to provide a more complete representation (than previously available) of the relationships between geometry and haemodynamics. The geometry is represented by a parametric CAD definition of a three-dimensional tuning-fork model of the human carotid artery bifurcation. The common carotid artery (CCA) bifurcates into two branches in the lower neck: the ICA and the ECA. Until relatively recently, many computer models of the carotid artery bifurcation have treated it as Y-shaped and thus neglected the significance of straightening the flow relative to the CCA. Ding et al. (2001) draw attention to the differences between the Y-shaped geometry and the so-called tuning-fork model. Interestingly, they claim that the Y-shaped geometry used by Bharadvaj et al. (1982) has become a standard in experimental and numerical investigations but they then proceed to show that a tuning-fork model represents a significant improvement, particularly in relation to the correlation between the oscillatory shear index (OSI) and the location of intimal thickness. Thomas et al. (2003) reinforce the preference for tuning-fork models especially for young healthy people.

Using a parametric CAD model facilitates systematic variation of the geometric parameters such that individual geometries can be automatically constructed and meshed in preparation for CFD simulation. Although significant time and effort is required to construct geometry and mesh models that can be repeatedly (and automatically) reused for a wide range of different shapes, the additional expense of automating this process is far less than that required to manually craft each geometry and mesh. Having constructed the parametric geometry model — and established a 
process for mesh construction and CFD simulation (including mesh and time dependence studies) - it is necessary to populate the design space of all feasible designs (or geometries). Techniques for doing this are commonly referred to as design of experiments (DoE), and a statistical $\mathrm{LP}_{\tau} \operatorname{DoE}$ (Sobol, 1979) is used here. Whilst the minimum DoE size for a certain level of accuracy is not normally known a priori, general guidance recommends using 10 times the number of variables. In order to visualise the relationships between geometry variables and $\tilde{\tau}$, a multi-dimensional surface is fitted to the objective function extracted from the CFD simulations performed in each geometry. Again, a range of methods are available for constructing such surfaces, and the method of kriging is used here (Keane and Nair, 2005).

\section{Problem definition}

\subsection{Parametric geometry model}

Careful consideration of a number of geometry descriptions including Bharadvaj et al. (1982), Ding et al. (2001) and Fanucci et al. (1988) shows that, from a CAD perspective, insufficient information is available in all of them to construct a parametric CAD model from scratch, without making a number of assumptions, and/or unrealistic constraints are imposed that limit the overall flexibility of the models. Consequently, a parametric CAD definition of the tuning-fork geometry is used as described in Bressloff et al. (2004). Using CATIA V5 (Dassault Systemes), the baseline model is adjusted simply by changing parameter values in an external file. The baseline geometry has mean dimensions taken from Bharadvaj et al. (1982), and is depicted in Fig. 1 and labelled with the parameters investigated here - the ICA and ECA angles $\left(\theta_{\mathrm{i}}\right.$ and $\theta_{\mathrm{e}}$, respectively), the ICA and ECA outflow diameters ( $\pi_{\mathrm{i}}$ and $\pi_{\mathrm{e}}$, respectively) and the position, $d$, of a Bezier curve control point, $P$, that controls the maximum width of the sinus bulb. The inner curve of the bulb is also constructed with a Bezier curve, but this is fixed relative to the line joining the start and end of the bulb. This has the effect of rotating the inner curve of the baseline shape. For the outer curve, the point $P$ is positioned by drawing a line from the point of maximum bulb width (point $F$ in Ding et al., 2001) and normal to the line joining the points defining the start of the outer ICA wall and the end of the sinus bulb. In this way, the Bezier curve that point $P$ controls is representative of the mean shapes characterised by Ding et al. (2001). Although, it is normal to directly vary the largest diameter of the bulb, the use of a Bezier curve is attractive since smooth curves can be generated by varying a single variable. Additionally, a check on the maximum diameter in all geometries shows that the maximum diameter of the sinus bulb is directly proportional to the value of $d$.

The two-dimensional definition shown in Fig. 1 is converted to a three-dimensional bifurcation by lofting

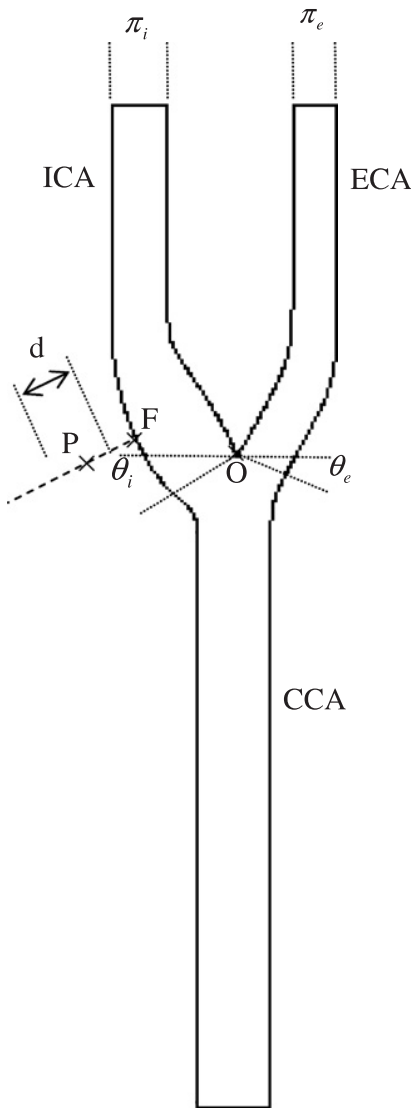

Fig. 1. Tuning-fork model.

surfaces through a set of circular planes drawn at suitable locations throughout the complete shape. The bounds of the design variables (based on the statistical data in Bharadvaj et al. (1982) such that a representative range of different geometries could be produced) are shown in Table 1 along with the values obtained for the baseline geometry (shape A) and those that generate the minimum (shape B) and maximum (shape C) values of $\tilde{\tau}$, and two other examples; one with a large bulb (shape D), and another with a small ICA angle (shape E) (Fig. 2).

\subsection{Computational mesh and CFD set-up}

Before committing to the exploration study, suitable mesh and time step sizes were determined using the baseline geometry and the following CFD set-up in Fluent 6.2.16 (Fluent Inc.). For this unsteady problem, the pressureimplicit with splitting of operators (PISO) pressure-velocity coupling scheme was used with second order accuracy in space and time. The pulsatile flow velocity profile was represented by a Fourier series of the waveform presented in figure 11 of Holdsworth et al. (1999), and the mass fluxes through the ICA and ECA volumes were fixed at 70:30.

Using time step sizes varying between 0.001 and $0.00001 \mathrm{~s}$, and a range of meshes from approximately 
Table 1

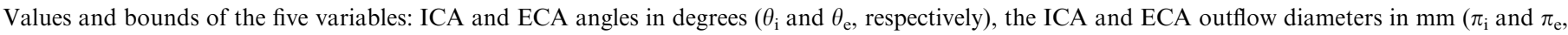
respectively) and the bulb width, $D$, in $\mathrm{mm}$ (controlled by varying the position, $d$, of the Bezier curve control point)

\begin{tabular}{|c|c|c|c|c|c|c|c|}
\hline & Upper & Lower & (A) Baseline & (B) Min. $\tilde{\tau}$ & (C) Max. $\tilde{\tau}$ & (D) & (E) \\
\hline$\theta_{\mathrm{e}}$ & 41.6 & 8.6 & 25.1 & 33.35 & 28.71 & 31.29 & 40.57 \\
\hline$\pi_{\mathrm{e}}$ & 6.6 & 2.6 & 4.6 & 5.7 & 5.4 & 3.4 & 3.3 \\
\hline$D$ & 12.4 & 8.0 & 10.2 & 9.1 & 12.2 & 12.2 & 8.1 \\
\hline$\tilde{\tau}$ & $\mathrm{N} / \mathrm{A}$ & $\mathrm{N} / \mathrm{A}$ & 0.232 & 0.131 & 0.343 & 0.262 & 0.155 \\
\hline
\end{tabular}

The units of $\tilde{\tau}$ and $A_{-}$are $\mathrm{Nm}^{-2}$ and $\mathrm{mm}^{2}$, respectively.

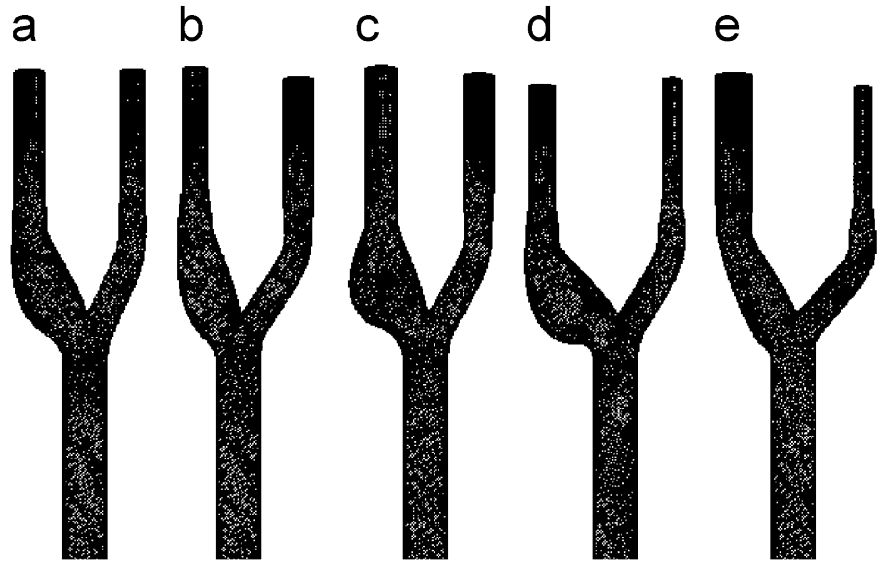

Fig. 2. Shape variation in the tuning-fork model. Bifurcations (a)-(e) are defined in Table 1.

46,000 to 4.4 million cells, only fractional differences were observed in velocity and shear stress profiles when the mesh spacing was reduced below $0.24 \mathrm{~mm}$ ( 1.3 million cells) and when the time step was below $0.0001 \mathrm{~s}$. Furthermore, these preliminary simulations also confirmed that two pulses were required to remove initial transients from the simulations. Using an AMD Opteron processor with a clock speed of $2.2 \mathrm{GHz}$, two pulses incorporating a $0.0001 \mathrm{~s}$ time step size on the $1.3 \mathrm{~m}$ cell mesh required $100 \mathrm{~h}$ run time. In light of the number of separate artery shapes to be considered, it was decided to perform the simulations using a mesh spacing of $0.48 \mathrm{~mm}$ (approximately 220,000 cells) which with two pulses required approximately $30 \mathrm{~h}$ of run time. Although there is no guarantee that the same conclusions will be reached when performing design studies that are identical in every way except the mesh resolutions, the values of $\tilde{\tau}$ were found to agree to within $8 \%$ between the 0.24 and $0.48 \mathrm{~mm}$ meshes when simulated in the bifurcation with maximum $\tilde{\tau}$. Furthermore, it is often found that relatively coarsely resolved (or lower fidelity) simulations can still capture trends in the objective function as design parameters are varied (Forrester, 2004).

\section{Results and discussion}

Having developed the baseline shape and configured a strategy for mesh construction and CFD simulation, it is necessary to select a set of shapes from which the variation of $\tilde{\tau}$ across the complete landscape can be predicted. A 50 point $\mathrm{LP}_{\tau}$ DoE is generated and, for each case, new geometries are constructed, meshes generated and the flow simulations performed using templates developed for the baseline shape.

To visualise the data generated from the DoE, a function (or hypersurface) is constructed through all points in the DoE using a stochastic process model (or krig) as described above. Preliminary inspection of the resulting hypersurfaces confirmed that the bulb width is the dominant variable. Consequently, it is convenient to visualise the multi-dimensional function by fixing the bulb width at specific values. Fig. 3 shows the variation of the other four variables at non-dimensional bulb widths characterised by (a) $d=0.0$, (b) $d=0.5$ and (c) $d=1.0$. These values correspond to maximum bulb diameters of 8.0, 10.2 and $12.4 \mathrm{~mm}$, respectively. Each sub-plot in Fig 3a shows the shape of the hypersurface for variations in the diameters of the ICA and ECA (as labelled in the top left sub-plot) for $d=0.0$ and various combinations of the ICA and ECA angles. The rows of sub-plots have $\theta_{\mathrm{e}}$ varying from 0.0 in the bottom row, through 0.5 in the middle row to 1.0 in the top row, whilst $\theta_{\mathrm{i}}$ is 0.0 in the left-hand column, 0.5 in the middle column and 1.0 in the right-hand column. All subsequent sub-plots have the same specification. The dimensional values of each parameter can be calculated from the upper and lower bounds listed in Table 1.

The main features to note in these plots, in addition to the strong dependence on bulb width, are that (i) the subplots in each sub-figure do not vary significantly, but (ii) at large bulb widths the response to variations in the outflow diameters is noticeably stronger than for small bulb widths.

The data used to generate these plots comprise the values of $\tilde{\tau}$ obtained at each of the 50 design points after two complete pulses. The objective function has been nondimensionalised on the maximum value from the data set. The reason for doing this is to compare the shape of the 
a
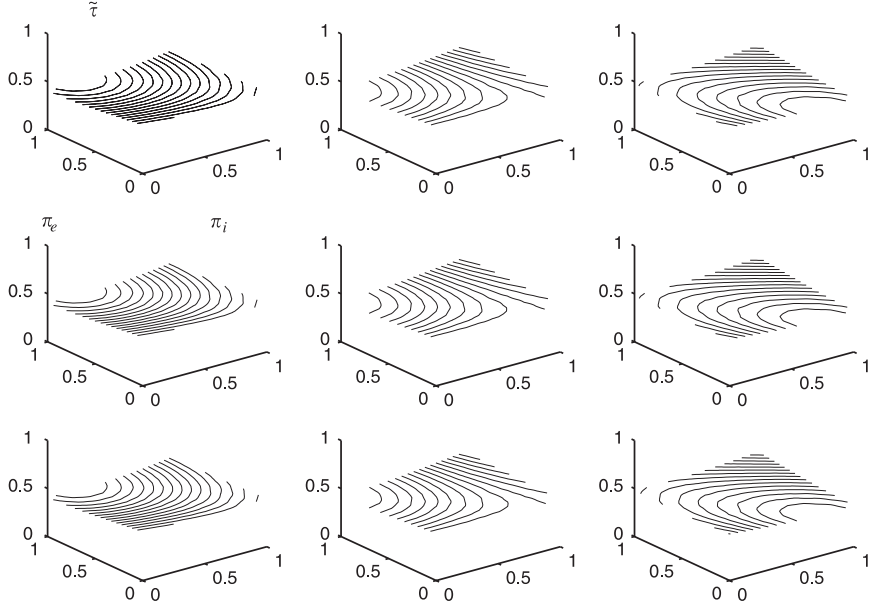

b
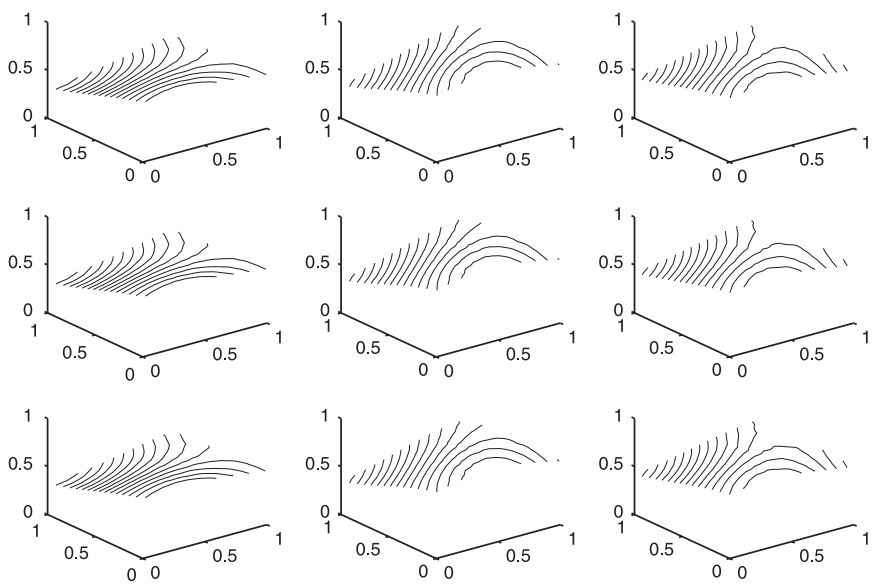

C
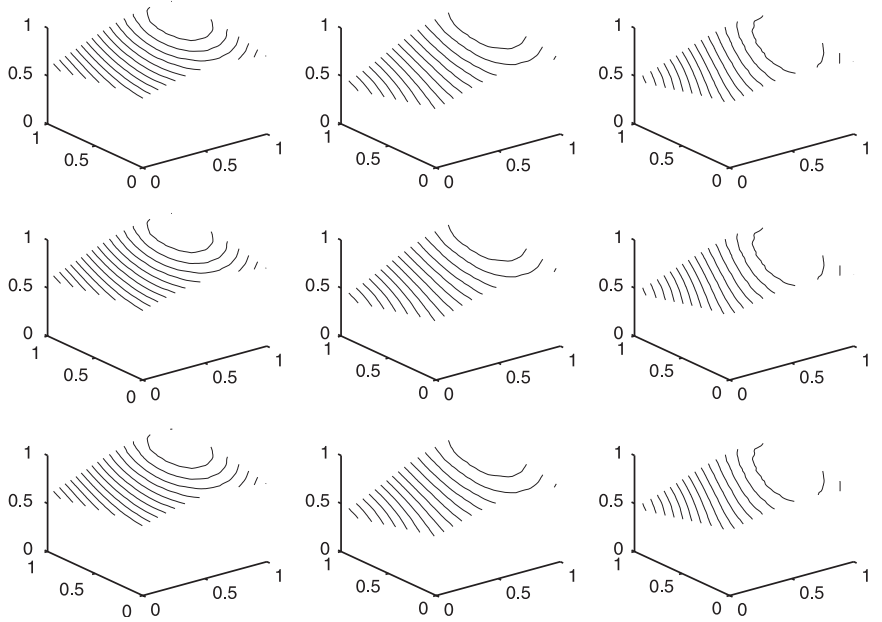

Fig. 3. Variation of non-dimensionalised $\tilde{\tau}$ at the end of the second pulse at various bulb widths: (a) minimum, (b) intermediate, (c) maximum. (Note: $\tilde{\tau}$ is non-dimensionalised by the maximum predicted value of $\tilde{\tau}$ at the end of the pulse.)

hypersurface at different times in the cycle. Similar plots produced after the first pulse (non-dimensionalised on the peak value at that time) are visually identical and, although there are quantitative differences in the objective function values (up to approximately $4 \%$ ), an evaluation of the variability between the surfaces equals 0.999 (3 d.p.) as measured by the Pearson product moment correlation coefficient,

$$
r=\frac{n\left(\sum A B\right)-\left(\sum A\right)\left(\sum B\right)}{\sqrt{\left[n\left(\sum A^{2}\right)-\left(\sum A\right)^{2}\right]\left[n\left(\sum B^{2}\right)-\left(\sum B\right)^{2}\right]}},
$$

where $A$ and $B$ denote, respectively, the arrays of equally spaced values of the kriging functions evaluated at the end of the two pulses. (Recall that initial transients have disappeared by the second pulse such that no variation is observed between the second and third pulses.)

Having compared the surrogate models in this way, the question as to the time in the cycle at which the hypersurface adopts its final shape effectively arose by chance. When running the above simulations, data was saved at the mid-points of each pulse as well as at the end of the two pulses for each bifurcation. It is common precautionary practice to autosave simulations at intermediate times in case of computer failure leading to wasted run time. Based on recent work that considered the evolution of response surfaces iterating towards steady state in aerodynamics problems (Forrester et al., 2006), a krig was constructed through the data for $\tilde{\tau}$ saved at the mid-point of the first pulse. Since this speculative construction revealed a model very similar in appearance to the models produced at the end of the two pulses, all 50 simulations were rerun for a single pulse, saving data at every 10 th of the cycle.

The evolution of the surrogate model is epitomised by the sub-plots depicted in Fig. 4 for maximum sinus bulb size (equivalent to Fig. 3c) at non-dimensional times 0.3, 0.4 and 0.5 in the pulse. In each sub-figure, $\tilde{\tau}$ is nondimensionalised by the maximum value at that time. Qualitatively, it can be seen that the sub-plots in Fig. 4c agree very closely with those in Fig. 3c, suggesting that only approximately half a pulse is needed to capture the shape of the hypersurface for a complete pulse.

Further evidence for the convergence of the shape of the hypersurface is shown in Figs. 5 and 6. The variation in $\tilde{\tau}$ through the pulse is depicted in Fig. 5 for the bifurcation shapes shown in Figs. 2a-e, defined in Table 1, and considered in further detail below. Although the values of $\tilde{\tau}$ do not converge asymptotically to a final value, it is clear that, following the peaks in each profile, the decreases in $\tilde{\tau}$ are far more gradual than the increase up to the peaks.

In Fig. 6, the Pearson product moment correlation coefficient is used to measure the extent of the agreement between surrogate models constructed at 0.1 intervals (i.e. every $0.0917 \mathrm{~s}$ ) through the first pulse relative to the surface at the end of the second pulse (cf. Fig. 3). In other words, at each particular time, values for $\tilde{\tau}$ are non-dimensionalised by the maximum value at that time, the kriging function is evaluated to construct a hypersurface, and a uniform mesh 
a
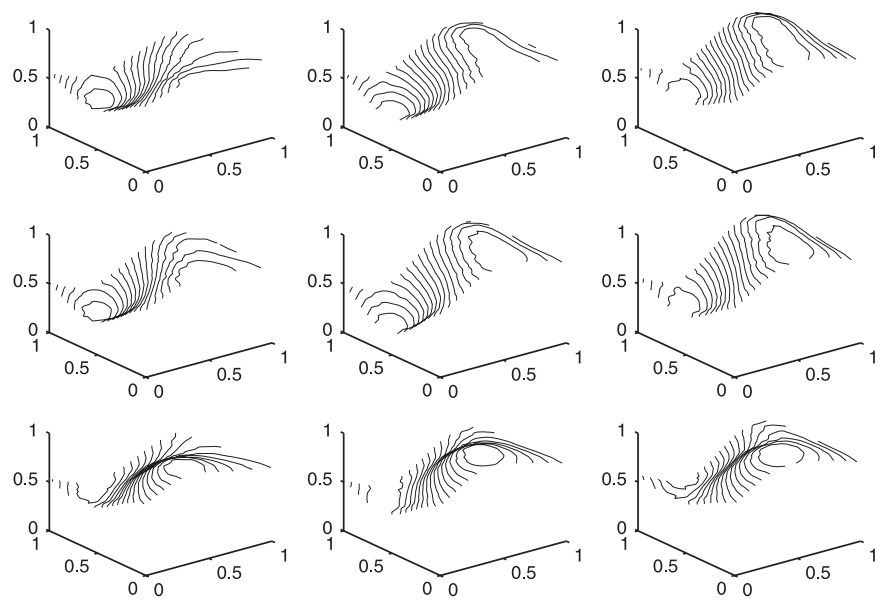

b
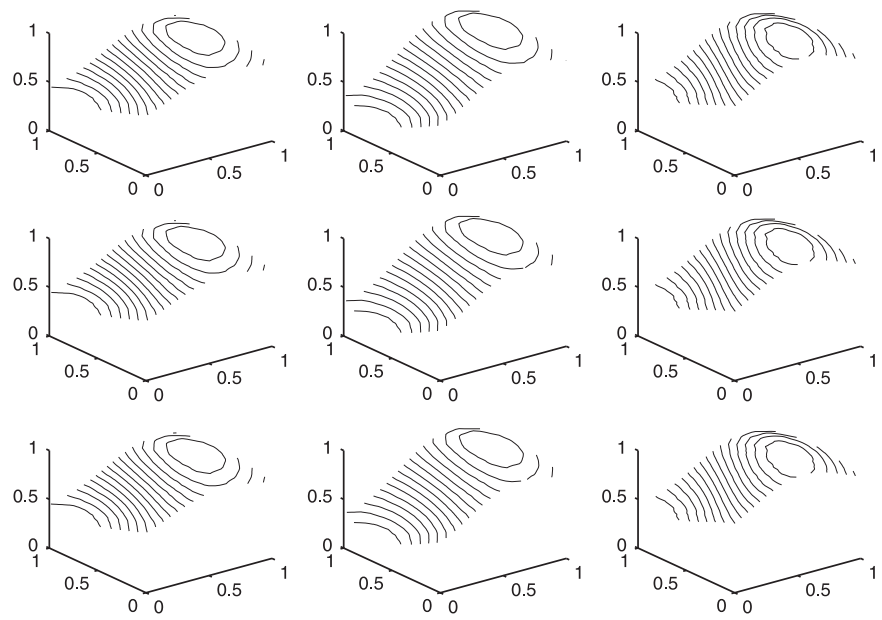

C
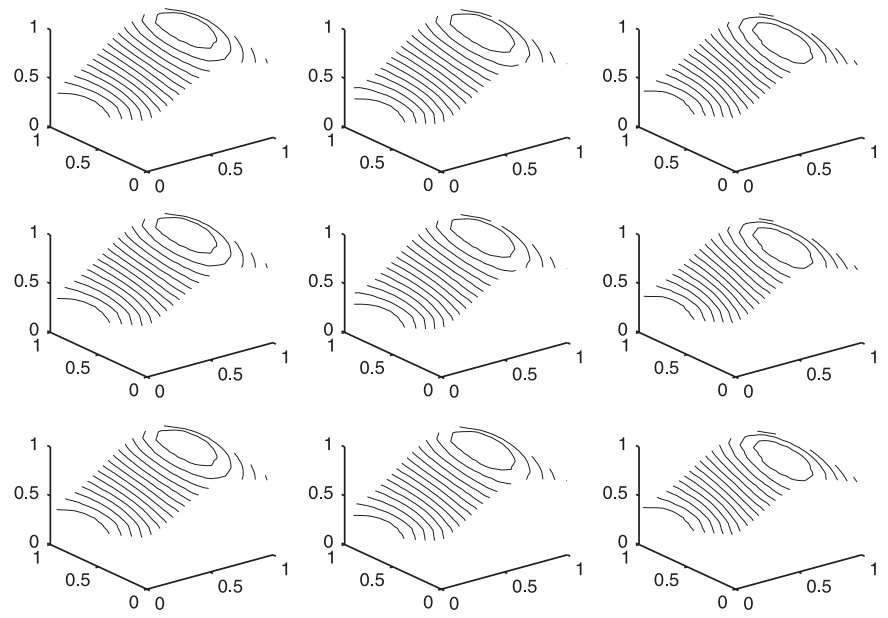

Fig. 4. Variation of non-dimensionalised $\tilde{\tau}$ for maximum bulb width at various non-dimensional times in the pulse: (a) 0.3 , (b) 0.4 , (c) 0.5. (Note: $\tilde{\tau}$ is non-dimensionalised in each plot by the maximum predicted value of $\tilde{\tau}$ at that time.) The sub-plot axis labels are the same as those in Fig. 3.

of $n$ points is used to extract the value of the prediction at those points, denoted by $B$ in Eq. (4). $r$ at that time is then evaluated by comparing these predictions with the equiva-

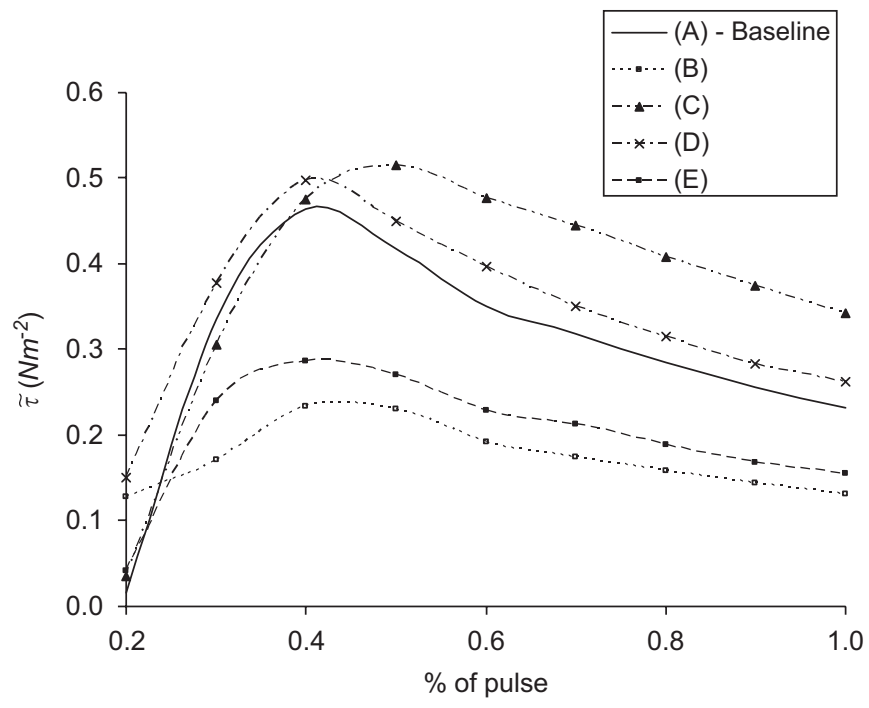

Fig. 5. Variation of $\tilde{\tau}\left(\mathrm{N} \mathrm{m}^{-2}\right)$ through the first pulse. See Fig. 2 and Table 1 for the bifurcation shapes (A)-(E).

lent set of predictions, $A$, from the kriging function created for $\tilde{\tau}$ at the end of the second pulse.

In Figs. 7 and 8 , the variation of $\bar{\tau}_{-}$is shown in the range 0 to $-0.315 \mathrm{~N} \mathrm{~m}^{-2}$. Fig. 7 depicts (for the geometry with maximum $\tilde{\tau}$ ) that the shape and extent of the bifurcation surface (for which $\bar{\tau}_{-}<0$ ) appears to converge at a slightly faster rate than the surrogate model. Comparing Fig. $7 \mathrm{~d}$ with Fig. $8 \mathrm{~b}$ (in which $\bar{\tau}_{-}<0$ is shown for the same geometry at the end of the first pulse) this region adopts a very close resemblance to its final shape after two-fifths of the pulse. Recall though that, as measured by the Pearson product moment correlation coefficient, slightly longer is needed for the shape of the surrogate model to reach a satisfactory level.

For shapes B (minimum $\tilde{\tau}$ ) and $\mathrm{C}$ (maximum $\tilde{\tau}$ ), the variation of $\bar{\tau}_{-}$at the end of the first pulse is shown in Figs. 8a and b, respectively. Unsurprisingly, the bulb size is the main difference between the two geometries, being close to the lower and upper limits, respectively. However, of the other variables, only the ICA outflow diameter, $\pi_{\mathfrak{i}}$, is significantly different, with the values of $\theta_{\mathrm{i}}, \theta_{\mathrm{e}}$ and $\pi_{\mathrm{e}}$ being very close for both geometries (cf. Table 1 ). In contrast to previous (limited) comparisons between bifurcation shapes, the multi-dimensional nature of this study suggests that interdependence between variables may have a significant effect on the flow, and the associated shear stress distribution. So, for example, with reference to Table 1, Fig. $8 \mathrm{c}$ shows a bifurcation with a large bulb and large ICA angle, but with relatively smaller outflow diameters than those in Fig. 8b; the two bifurcations have similar ECA angles. With this combination of parameters, $\tilde{\tau}$ is nearly $25 \%$ lower than that for the bifurcation with maximum $\tilde{\tau}$, and only marginally higher than that for the baseline shape. From a visual comparison of Figs. $8 \mathrm{~b}$ and c, it appears that the areas are broadly similar over which $\bar{\tau}_{-}<0$. However, numerically, the area in Fig. $8 \mathrm{~b}$ is 


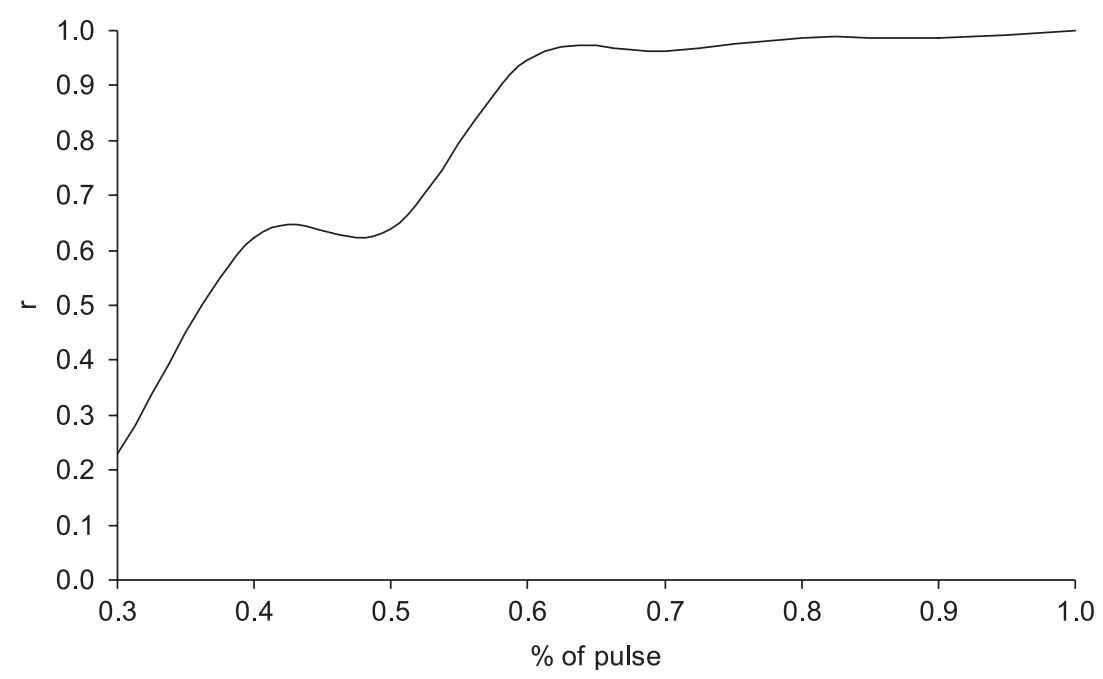

Fig. 6. Variation of the Pearson product moment correlation coefficient $r$ (Eq. (4)) with \% of pulse.

a

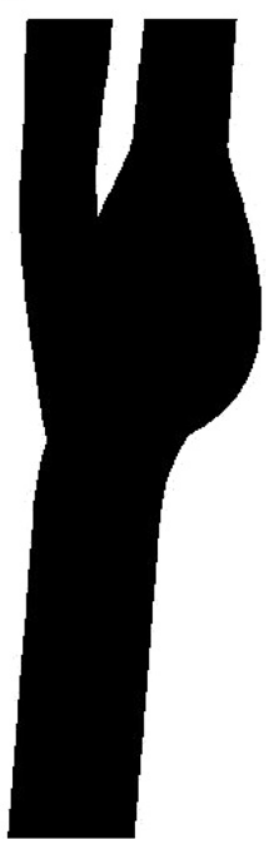

b

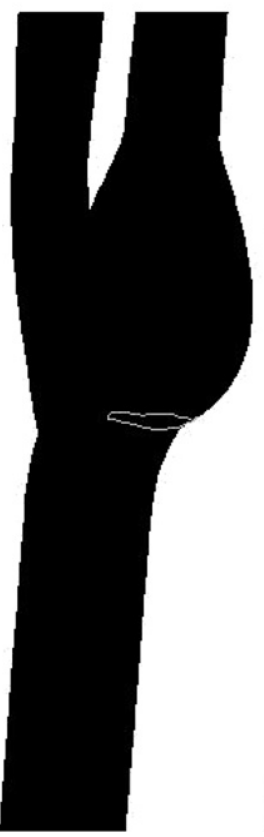

C

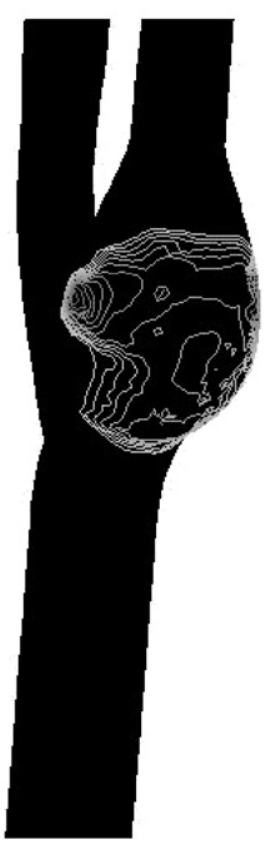

d

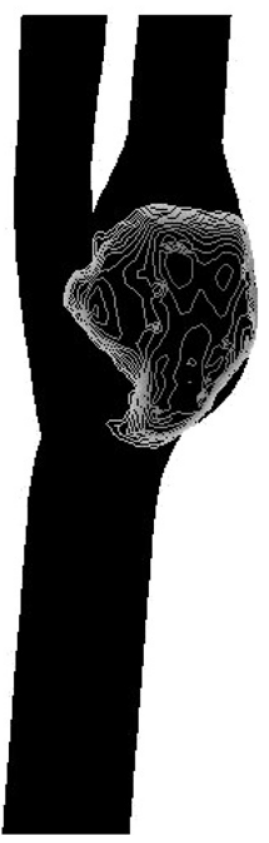

Fig. 7. Variation of $\bar{\tau}_{-}$(between 0 and $-0.315 \mathrm{~N} \mathrm{~m}^{-2}$ ) in bifurcation shape (C) with maximum $\tilde{\tau}$ at various times in the pulse: (a) 0.1 , (b) 0.2 , (c) 0.3 , (d) 0.4 .

approximately $15 \%$ larger than that in Fig. $8 \mathrm{c}$, and the $25 \%$ discrepancy in $\tilde{\tau}$ is dominated by a $30 \%$ difference in the non-normalised value of $\tilde{\tau}$ (i.e. the numerator in Eq. (1)). It is clear from this discussion that two different metrics are effectively under scrutiny: the strength and the extent of $\bar{\tau}_{-}$, the latter being given by the area expression in the denominator of Eq. (1). Interestingly, whereas it might be expected that there would be a strong positive correlation between the values of $\tilde{\tau}$ and the area, $A_{-}$, for which $\bar{\tau}_{-}<0$, this hypothesis is confounded by the evidence presented so far (viz., the area depicted in Fig. 8a is only marginally smaller than that in Fig. 8c), and is further clouded by the distribution shown in Fig. 8d. This bifurcation most notably has a very small bulb width (and other parameter settings as tabulated in Table 1), and has the lowest value of $A_{-}$in all of the 50 bifurcations considered. Although, relative to Fig. 8a, $\mathrm{A}_{-}$is a factor of three lower in Fig. 8d, $\tilde{\tau}$ agrees to within $10 \%$ due to the more intense level of $\bar{\tau}_{-}$in Fig. 8d.

Whereas earlier studies have attempted to identify correlations between individual geometric parameters and shear stress metrics of interest, the comparisons considered here suggest that a far more complex set of relationships are interacting to determine the distribution and extent of shear stress behaviour - certainly as measured by $\bar{\tau}_{-}, A_{-}$ and $\tilde{\tau}$. In terms of ranking geometry, it would not appear to 
a

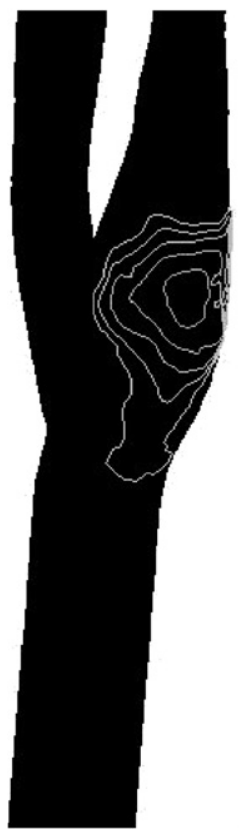

b

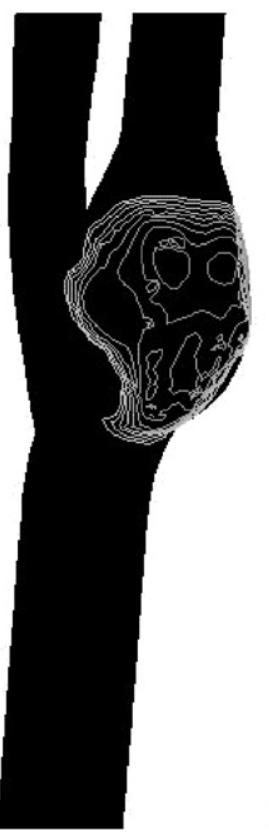

C

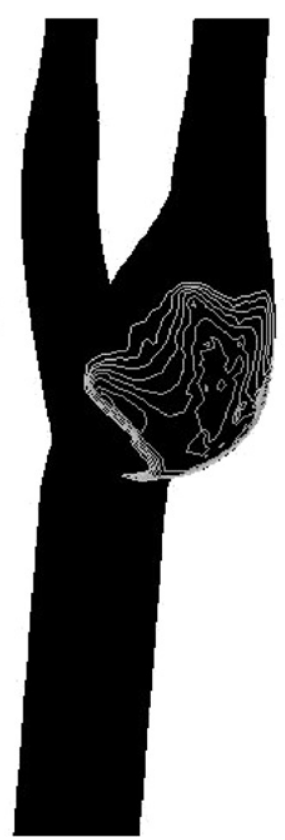

d

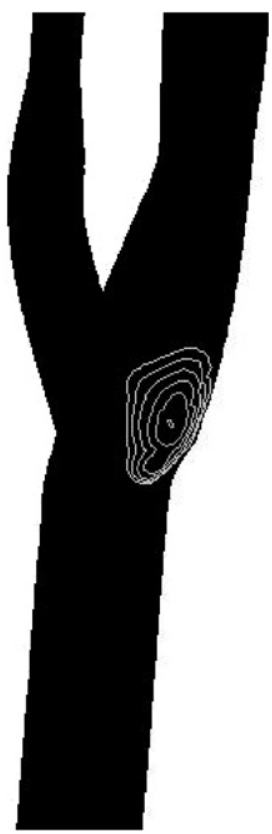

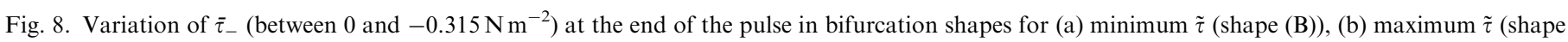
(C)), (c) large ICA angle (shape (D)), (d) small bulb width (shape (E)).

be sufficient to simply claim that a particular parameter should be as large or as small as possible. Qualification should even be applied to the bulb width, for which the strongest correlation with recirculating flow appears to exist. For example, it has been demonstrated here that a relatively small bulb width (cf. Fig. 8d) may produce a significantly smaller but more intense region of reverse flow than a larger bulb (cf. Fig. 8a) - with different settings of other parameters such as the ICA and ECA angles and outflow diameters. However, the two bifurcations yield broadly similar values (within 10\%) for the shear stress metric, $\tilde{\tau}$.

\section{Conclusions}

Pulsatile flow through a three-dimensional computational model of the carotid artery bifurcation has been used to demonstrate how a surrogate model can be constructed to represent the relationships between multiple geometric parameters and flow metrics of interest. The landscape so constructed can then be explored to identify regions of good or bad shapes, or to assess how the metrics are affected by manipulation of one or more of the parameters.

This approach is dependent upon the credibility of the metrics employed to measure the performance of different shapes. Although there is a consensus view concerning the correlation between regions of recirculation and sites of disease in the carotid artery bifurcation, the evidence is inconclusive. Also, as shown here, more than one metric may have an effect, and it is not clear whether the intensity and/or the extent of the effect are both (equally) important, or whether other metrics such as the peak values or the duration for which the metric is above a certain threshold are significant. Further evidence is needed to define how endothelial cells respond to these different metrics. Notwithstanding this uncertainty, the analysis presented here has confirmed that the width of the sinus bulb is the dominant variable of those considered, and that a larger bulb width generally produces higher values of the areaweighted integral of time-averaged shear stress, $\tilde{\tau}$. Conversely, low values of $\tilde{\tau}$ are associated with smaller bulb widths, but these can be produced by different combinations of the ICA and ECA flow angles and outflow diameters, leading to either small, more intense regions or large weaker regions of reverse flow.

Most significantly, this work demonstrates that only approximately half a single pulse is required for the shape of the surrogate model to converge to within a few percent of its final pulse-independent shape. Thus, if expensive CFD simulations are to be used in clinical planning, an extensive comparison between geometries can be accomplished by the surrogate modelling approach presented here benefiting from a significant time saving afforded by the need to only simulate a fraction of a single pulse. Although alternative haemodynamic parameters may be used in future studies, it is to be hoped that early convergence of their associated response surfaces could be exploited in a similar way.

\section{Acknowledgements}

The author would like to acknowledge the input from Jim Banks and Vijaya Kolachalama Bhaskar, who were both very much involved with the construction of the 
parametric model used in this research. Also, Professor Cliff Shearman has provided inspirational discussions into the potential clinical relevance of this work.

\section{References}

Augst, A.D., Barratt, D.C., Hughes, A.D., Thom, S.A.M., Xu, X.Y., 2003. Various issues relating to computational fluid dynamics simulations of carotid bifurcation flow based on models reconstructed from three-dimensional ultrasound images. Proceedings of the Institution of Mechanical Engineers, Part H-Journal of Engineering in Medicine 271 (5), 393-403.

Bharadvaj, B.K., Mabon, R.F., Giddens, D.P., 1982. Steady flow in a model of the human carotid bifurcation. Part I-Flow visualisation. Journal of Biomechanics 15 (5), 349-362.

Bhaskar, K.V., Bressloff, N.W., Nair, P.B., Shearman, C.P., 2005. A parametric study of the hemodynamic behavior in a one dimensional systemic elastic artery: application to stent design. In: 12th International Conference on BioMedical Engineering (ICBME 2005).

Bressloff, N.W., Banks, J., Bhaskar, K.V., 2004. Parametric geometry definition and analysis of the human carotid artery bifurcation. IMECE2004-59124. In: ASME Mechanical Engineering Congress, Anaheim, CA.

Bressloff, N.W., Banks, J., Shearman, C.P., 2005. Systematic exploration into the hemodynamic effect of an out-of-plane internal carotid artery. In: 2005 Bioengineering Conference, ASME 2005.

Buchanan Jr., J.R., Kleinstreuer, C., 1998. Simulation of particlehemodynamics in a partially occluded artery segment with implications to the initiation of microemboli and secondary stenoses. Journal of Biomechanical Engineering, Transactions of the ASME 120, 446-454.

Ding, Z., Wang, K., Li, J., Cong, X., 2001. Flow field and oscillatory shear stress in a tuning-fork-shaped model of the average human carotid bifurcation. Journal of Biomechanics 34, 1555-1562.

Fanucci, E., Orlacchio, A., Pocek, M., 1988. The vascular geometry of human arterial bifurcations. Investigative Radiology 23 (10), 713-718.

Forrester, A.I.J., 2004. Efficient global aerodynamic optimisation using expensive computational fluid dynamics simulations. PhD. Thesis, University of Southampton, UK.

Forrester, A.I.J., Bressloff, N.W., Keane, A.J., 2006. Optimisation using surrogate models and partially converged computational fluid dynamics simulations. Proceedings of the Royal Society 462, 2177-2204.
Holdsworth, D.W., Norley, C.J.D., Frayne, R., Steinman, D.A., Rutt, B.K., 1999. Characterization of common carotid artery blood-flow waveforms in normal human subjects. Physiological Measurements 20, 219-240.

Hyun, S., Kleinstreuer, C., Longest, P.W., Chen, C., 2004. Particle hemodynamics simulations and design options for surgical reconstructions of diseased carotid artery bifurcations. Journal of Biomechanical Engineering, Transactions of the ASME 126, 188-195.

Keane, A.J., Nair, P.B., 2005. Computational Approaches for Aerospace Design: the Pursuit of Excellence. Wiley, New York.

Ku, D.N., Giddens, D.P., Zairns, C.K., Glagov, S., 1985. Pulsatile flow and atherosclerosis in the human carotid bifurcation. Arteriosclerosis 5 (3), 293-302.

Milner, J.S., Moore, J.A., Rutt, B.K., Steinman, D.A., 1998. Hemodynamics of human carotid artery bifurcations: computational studies with models constructed from magnetic resonance imaging of normal subjects. Journal of Vascular Surgery 28 (1), 143-156.

Perktold, K., Peter, R.O., Resch, M., Langs, G., 1991. Pulsatile nonNewtonian blood flow in three-dimensional carotid bifurcation models: a numerical study of flow phenomena under different bifurcation angles. Journal of Biomedical Engineering 13, 507-515.

Schulz, U.G.R., Rothwell, P.M., 2001. Sex differences in carotid bifurcation anatomy and the distribution of atherosclerotic plaque. Stroke 32, 1525-1531.

Sitzer, M., Puac, D., Buehler, A., Steckler, D.A., von Kegler, S., Markus, H.S., Steinmetz, H., 2003. Internal carotid artery angle of origina novel risk factor for early carotid atherosclerosis. Stroke 34 (4), 950-955.

Sobol, I.M., 1979. On the systematic search in a hypercube. SIAM Journal on Numerical Analysis 16, 790-793.

Spelde, A.G.A., de Vos, R.A.I., Hoogendam, I.J., Heethar, R.M., 1990. Pathological-anatomical study concerning the geometry and atherosclerosis of the carotid bifurcation. European Journal of Vascular Surgery 4, 345-348.

Thomas, J.B., Che, S.L., Milner, J.S., Antiga, L., Rutt, B.K., Spence, J.D., Steinman, D.A., 2003. In: Summer Bioengineering Conference, Florida, USA.

Wells, D.S., Archie, J.P., Kleinstreuer, C., 1996. Effect of carotid artery geometry on the magnitude and distribution of wall shear stress gradients. Journal of Vascular Surgery, 667-678.

Zairns, C.K., Giddens, D.P., Bharadvaj, B.K., Sottiurai, V.S., Mabon, R.F., Glagov, S., 1983. Carotid bifurcation atherosclerosis. Circulation Research 53 (4), 502-514. 